\title{
7-T MRI of explanted liver and ex-vivo pancreatic specimens: prospective study protocol of radiological-pathological correlation feasibility (the EXLIPSE project)
}

Rosa Cervelli ${ }^{1}$, Matteo Cencini ${ }^{2}$, Guido Buonincontri ${ }^{2}$, Francesco Campana ${ }^{1}$, Andrea Cacciato Insilla ${ }^{3}$, Giacomo Aringhieri ${ }^{1}$, Paolo De Simone ${ }^{4}$, Ugo Boggi ${ }^{5}$, Daniela Campani ${ }^{3}$, Michela Tosetti ${ }^{2}$ and Laura Crocetti ${ }^{1 *}$ (D)

\begin{abstract}
The study focuses on radiological-pathological correlation between imaging of ex vivo samples obtained by a 7-T scanner and histological examination. The specimens will be derived from native explanted cirrhotic livers, liver grafts excluded from donation because of severe steatosis, and primary pancreatic tumours. Magnetic resonance imaging (MRI) examinations will be performed within $24 \mathrm{~h}$ from liver or pancreatic lesion surgical removal. The MRI protocol will include morphological sequences, quantitative T1, T2, and fat-, water-fraction maps with Cartesian k-space acquisition, and multiparametric methods based on a transient-state "MRI fingerprinting". Finally, the specimen will be fixed by formalin. Qualitative imaging analysis will be performed by two independent blinded radiologists to assess image consistency score. Quantitative analysis will be performed by drawing regions of interest on different tissue zones to measure $\mathrm{T} 1$ and $\mathrm{T} 2$ relaxation times as well as fat- and water-fraction. The same tissue areas will be analysed by the pathologists. This study will provide the possibility to improve our knowledge about qualitative and quantitative abdominal imaging assessment at 7T, by correlating imaging characteristics and the corresponding histological composition of ex vivo specimens, in order to identify imaging biomarkers. Trial registration: ClinicalTrials.gov: 13646. Registered 9 July 2019-retrospectively registered
\end{abstract}

Keywords: Liver cirrhosis, Liver steatosis, Liver transplantation, Magnetic resonance imaging, Pancreatic neoplasms

\section{Key points}

- Explanted livers and pancreatic specimens will be investigated by 7-T magnetic resonance imaging (MRI).

- Morphological findings and quantitative data will be compared with specimen histology.

- Quantitative MRI will be explored for liver and pancreatic lesion characterisation.

\footnotetext{
* Correspondence: laura.crocetti@med.unipi.it

'Division of Diagnostic and Interventional Radiology, University of Pisa, Via

Paradisa, 2 - Cisanello Hospital, 56100 Pisa, Italy

Full list of author information is available at the end of the article
}

- MRI fingerprinting will be used to estimate quantitative maps in a shorter time.

\begin{abstract}
Background
Magnetic resonance imaging (MRI) provides information on tissue structure and function by means of measurements of intrinsic differences in relaxation times of hydrogen (as well as other) nuclei following radiofrequency excitation. By increasing the magnetic field of the equipment, an intrinsic increase of the signal-to-noise ratio can be achieved $[1,2]$. As a consequence, ultra-high magnetic field strengths such as that used in 7-T units may provide clinical advantages by
\end{abstract}


improving quantitative map estimation, increasing the spatial resolution, or reducing the scan time.

This study focuses on the comparison between 7-T MRI of ex vivo samples and their histological examination, to assess the correlation between tissue imaging characteristics and pathological features. The examined samples will be native cirrhotic livers explanted during orthotopic liver transplantation (group A), liver grafts excluded from donation because of severe steatosis (group B), and surgical specimens of primary pancreatic tumours, which underwent upfront pancreaticoduodenectomy or total pancreatectomy (group C). To obtain the information on tissue structure and function, this study will include conventional methods and novel multiparametric quantitative evaluation. The novel multiparametric methods are based on a transient-state magnetic resonance fingerprinting (MRF) [3] sequence with spiral trajectory $k$-space acquisitions in two or three dimensions. While conventional imaging for quantitative mapping usually needs multiple acquisitions to obtain the measurements of the different parameters, MRF can provide multiple quantitative information with a single acquisition. This technique compares the variable signal acquired per each pixel during the acquisition with a predefined dictionary of signal evolution, allowing the recognition and the measurement of the different parameters based on the match of their signal evolution. Using MRF to evaluate the upper abdomen, including the liver and pancreas, Chen et al. [4] succeeded in the simultaneous quantification of $\mathrm{T} 1$ and $\mathrm{T} 2$ relaxation time values in the abdomen with a 19-s breath-hold acquisition.

The radiological-pathological correlation may improve the knowledge of the changes that occur in cirrhotic liver and their focal lesions (inflammatory, proliferative non-neoplastic, degenerative, and neoplastic lesions), in non-alcoholic steatotic liver disease, which represents one of the main cause of unsuitability for liver transplant liver graft, and pancreatic ductal adenocarcinoma (PDAC, group C). More in detail, the T1 and T2 relaxation time values and the fat fraction will be assessed on targeted tissues, consisting of focal pancreatic/hepatic lesions or liver parenchyma (for the excluded liver grafts) and of parenchymal tissues different from the targeted one such as non-neoplastic surrounding parenchyma (non-targeted tissues).

We assume that statistically significant differences in relaxation time values between the lesions and non-targeted tissue will be observed.

In fact, as already explored in different clinical fields, $\mathrm{T} 1$ and $\mathrm{T} 2$ relaxation time estimation provided promising results in oncologic and non-oncologic tissue characterisation and evaluation of neoplastic tissue changes after treatment [5]. Among these applications, quantitative MRI has been shown to be useful in the evaluation of tissue compositional changes occurring in liver fibrosis and hemosiderosis [6-8], as well as in the assessment of the liver fat content in the steatosis and steatohepatitis scenarios $[8,9]$. Similarly, pancreatic parenchyma have been studied through quantitative MRI analysis in both acute and chronic pathological processes with promising identification of noninvasive imaging biomarkers, including $\mathrm{T} 1$ and $\mathrm{T} 2$ relaxation time values [10]. Further studies are needed to explore the role of quantitative MRI in understanding the underlying pathological tissue changes and processes.

The rationale behind the group A of native livers explanted from patients undergoing orthotopic liver transplantation derives from the possibility of investigating focal lesions focusing on hepatocellular carcinoma $[11,12]$ and the effect of locoregional therapy (microwave ablation, transarterial chemoembolisation, and ${ }^{90} \mathrm{Y}$ transarterial radioembolisation), when applied as a "bridge treatment" [13]. All this information will be collected into a data bank to improve the knowledge of many conditions that affect liver candidates for transplant.

Group B will include grafts that are excluded from liver transplantation due to severe macrovesicular steatosis $[14,15]$. Due to the limited availability of organs for liver transplant and long waiting lists for transplantation, many attempts were made to increase the number of donors, including the use of the "steatotic extended criteria donor" [16]. The risk of graft failure and dysfunction after transplantation of those livers is related to an increase risk in ischemia-reperfusion injury [17]. Thus, the group B of livers represent precious material to investigate the distribution of steatosis and the liver damage induced by it, in order to identify and select "good-steatotic livers" by 7-T MRI.

Finally, regarding PDAC, despite significant improvements in surgical and nonsurgical treatment modalities, including new possible therapeutic targets $[18,19]$, 5 -year survival rate of $5 \%$ of this tumour remains almost unchanged. Therefore, reproducible preclinical models are required to study the growth pattern and local peritumoural environment of pancreatic cancer, to develop new and effective treatment modalities for in vivo applications. These considerations guide the aim of the study for group C: the study of ex vivo pancreatic surgical specimens by 7-T MRI should provide a reference framework to define proper acquisition protocols and imaging biomarkers for future clinical applications.

\section{Study aims}

The main aim is to evaluate the correlation between the characteristics detected by the 7-T equipment and the histological composition of ex vivo specimens. 
The primary study aims for groups A and B are to identify useful imaging biomarkers based on the evaluation of:

- Radiological-pathological correlation of focal lesions affecting the cirrhotic liver and of fibrotic/regenerative characteristics of the liver tissue, free from focal lesions; comparisons will include morphological matching as well as correlations between T1 and T2 relaxation time values and differential diagnosis between lesions and pseudolesions or histological classification of the fibrosis degree;

- Radiological-histological correlation of hepatic lesions treated by locoregional "bridge" treatments, in particular by evaluating the $\mathrm{T} 1$ or $\mathrm{T} 2$ relaxation time value difference between lesion histologically classified as complete responders and as partial responder after treatment;

- The degree of steatosis and to correlate this radiological grading with the well-established histological classification of the steatosis distribution [20];

The study aim for group $\mathrm{C}$ is the following:

- Radiological-pathological correlation of pancreatic lesions; comparisons will include morphological matching between macroscopic histological evaluation and MRI as well as the correlations between $\mathrm{T} 1$ and $\mathrm{T} 2$ relaxation time values with the lesion extracellular microenvironment and lesion aggressiveness, respectively.

Secondary study aim is to standardise and validate new 7-T MRI protocols, including quantitative sequences, to be possibly implemented also on 3-T clinical scanners.

\section{Methods}

This research protocol is a prospective experimental correlational no-profit study on human ex vivo specimens from liver and pancreatic tissues.

\section{Ethical issues and enrolment}

Before starting the study, every participant of A and C groups will be accurately informed about the management of the resected specimen (whole native liver explanted during orthotopic liver transplantation or pancreatic specimens resected due to the presence of a PDAC lesion) and will be given the informative letter for them and for their general practitioner, the forms of the informed consent, and the one for the treatment of their data for research purposes. All participants will be registered after signing the informed consent. The patients' eligibility criteria, based on the study group of enrolment, are summarised in Table 1. The informed consent to participate to the "EXLIPSE" research study does not apply to group $\mathrm{B}$ since the authorisation to organ donation in our country implies also the consent to investigate the explanted organs for scientific purposes.

The study will be performed in agreement with the dictates of the Helsinki Declaration and the rules of Good Clinical Practice (E6: Good Clinical Practice: Consolidated Guideline (CPMP/ICH/135/95)). The approval of regional Ethical Committee to conduct this "single-centre prospective study" was obtained (protocol number approval: 13646).

The enrolment stage, as well as the analysis duration, will last 3 years.

\section{7-T MRI}

It is well established by the literature that an unavoidable signal difference between ex vivo and in vivo biological tissues is the one caused by fixation [21, 22]. In fact, fixation reduces both relaxation times and diffusivity. Thus, the specimens will be placed in a sterile, nonpyrogenic solution for hypothermic storage (Servator $\mathrm{C}^{\bullet}$, SALF, Bergamo, Italy). Each specimen will be maintained at a temperature ranging between 4 and $8{ }^{\circ} \mathrm{C}$ in the solution, until the MRI examination is started.

During the MRI study, the specimen will be placed in a dedicated single-use plastic box previously disinfected by a $90 \%$ alcohol solution and dried by a sterile towel (Supplemental material, Fig. S1). The MRI acquisition protocol will start when the specimen reaches the temperature of $20^{\circ} \mathrm{C}$ (in balance with the constantly monitored room temperature); that temperature increase allows the hydrogen-proton, positively charged, to turn its spin axis in order to generate the resonance signal phenomena. During the MRI examination, liver or pancreatic specimen temperature will range from 20 to $25^{\circ} \mathrm{C}$; if an over-range increase takes place, the examination will be interrupted. The temperature increase will be verified and monitored by the real-time estimation of specific absorption rate provided by the scanner, which allows the indirect estimation of the temperature increase. In addition, a MRI-compatible fibreoptic temperature probe will be used at the end of each sequence as further confirmation that the temperature limits are respected. Finally, the pathologist will identify any sign of specimen degeneration/contamination. Specimens with pathological signs of degeneration/ contamination will be excluded.

To guarantee the correct storage of the specimens, so that they maintain all the physiological, histological characteristics, all the MRI examinations will be performed within $24 \mathrm{~h}$ from the liver or pancreatic lesion surgical removal. At the end of the MRI study, the ex vivo specimens will be again stored in the solution at $4{ }^{\circ} \mathrm{C}$. Finally, the native explanted liver, the liver graft, 
Table 1 Inclusion and exclusion criteria according to the sample group

\begin{tabular}{|c|c|c|}
\hline & Inclusion criteria & Exclusion criteria \\
\hline Group " $A$ " of native liver & $\begin{array}{l}\text { - Age } \geq 18 \text { years } \\
\text { - Informed consent } \\
\text { - Full possession of one's own faculties } \\
\text { - Patients who are going to undergo liver transplantation, } \\
\text { independently from previous therapy or from the } \\
\text { primary disease they are affected by }\end{array}$ & $\begin{array}{l}\text { - Patients affected by polycystic liver disease } \\
\text { - Excluded from liver transplantation }\end{array}$ \\
\hline Group "B" of excluded grafts & $\begin{array}{l}\text { - Liver grafts with histologically proven macrovesicular } \\
\text { steatosis, not fit for transplantation }\end{array}$ & $\begin{array}{l}\text { - Liver grafts histologically proven macrovesicular } \\
\text { steatosis fit for transplantation } \\
\text { - Liver grafts not fit for transplantation due to } \\
\text { causes other than steatosis }\end{array}$ \\
\hline Group "C" of focal pancreatic lesions & $\begin{array}{l}\text { - Age } \geq 18 \text { years } \\
\text { - Informed consent } \\
\text { - Full possession of one's own faculties } \\
\text { - Affected by pancreatic lesion histologically proven } \\
\text { to be a carcinoma or with imaging and laboratory } \\
\text { findings highly suspicious for PDAC, and treated } \\
\text { by up-front pancreaticoduodenectomy or total } \\
\text { pancreatectomy } \\
\text { - Assessed by contrast-enhanced CT examination } \\
\text { within } 21 \text { days before surgery }\end{array}$ & $\begin{array}{l}\text { - Excluded from surgery due to the severity of their } \\
\text { condition. }\end{array}$ \\
\hline
\end{tabular}

and/or the primary pancreatic lesions will be fixed by formalin and analysed by the pathologist.

The imaging protocol will include both morphological and quantitative imaging acquisitions performed on a Discovery MR 950 7-T MRI scanner (General Electric Healthcare, Milwaukee - WI, USA) equipped with an autonomously customised 8-channel transmit/receive knee coil.

Morphological acquisitions They will include a threedimensional CUBE T2-weighted fast turbo spin-echo sequence as anatomical reference, as well as T1- and T2weighted water-only sequences using the "iterative decomposition of water and fat with echo asymmetry and least-squares estimation" (IDEAL) technique [23] and two- and three-dimensional cholangiopancreatography acquisitions. Imaging parameters are listed in Table 2.

Quantitative mapping will include the following series of techniques and methods.

T1 mapping T1 values will be obtained using a modified magnetisation-prepared rapid gradient echo acquisition, named MP2RAGE. Briefly, it consists of the combination of two low-flip angle images with different inversion times (TI). These images are combined according to the following equation

$$
\text { MP2RAGE intensity }=\frac{\mathrm{TI}_{1} \mathrm{TI}_{2}}{\mathrm{TI}_{1}^{2}+\mathrm{TI}_{2}^{2}}
$$

where $\mathrm{TI}_{1,2}$ are the two images with different $\mathrm{TIs}\left(\mathrm{TI}_{1}\right.$ and $\mathrm{TI}_{2}$ ). This combined image, due to the low flip angle, results to be bias corrected. Moreover, exploiting the knowledge of the acquisition parameters, the $\mathrm{T} 1$ values can be uniquely determined from the intensity values as previously described [24].

T2 mapping T2 values will be obtained by multi-echo spin-echo acquisition. Images for the individual echoes are used to obtain the T2 map using the following signal model:

$$
\ln (S(\mathrm{TE}))=\ln \left(S_{0}\right)-\frac{\mathrm{TE}}{T_{2}}
$$

where $S(\mathrm{TE})$ represents the voxel-wise signal magnitude for the echo time (TE).

Fat fraction mapping Fat fraction values will be obtained from an IDEAL fast gradient-echo acquisition. To avoid T1-weighting and obtain a proton density-weighted fat fraction, a low flip angle is used. Output water and fat images are combined to obtain fat fraction map, as follows:

$$
\mathrm{FF}=\frac{F}{W+F}
$$

where $F$ and $W$ represent the signal of fat and water, respectively.

Multiparametric techniques (MRF) Multiparametric acquisitions in the transient state (i.e., preventing the magnetisation from reaching an equilibrium condition by continuously changing the acquisition parameters between each readout) will be included in the protocol. Two different MRF acquisitions will be performed: a threedimensional acquisition based on spiral projections, which will provide $\mathrm{T} 1, \mathrm{~T} 2$, and $\mathrm{B}_{1}+$ transmit field maps, and a 


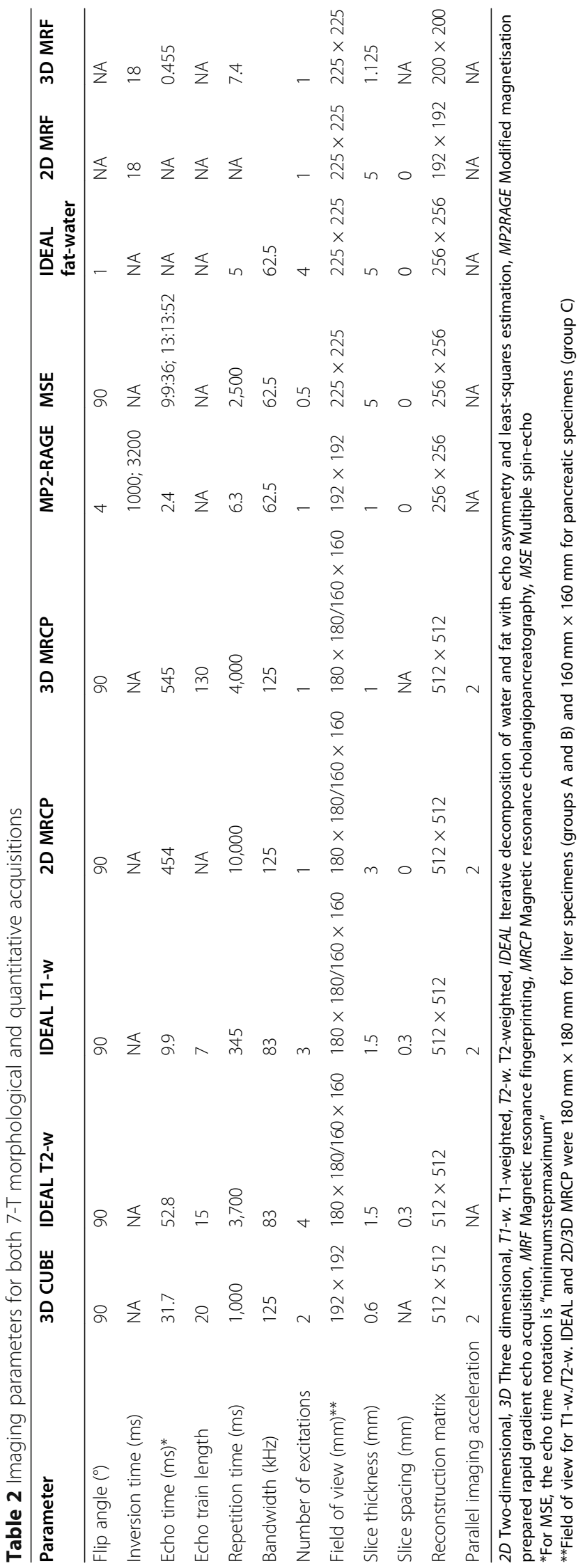


spiral two-dimensional acquisition, which will provide T1, $\mathrm{FF}, \mathrm{B}_{0}$, and $\mathrm{B}_{1}+$ transmit field maps. In both cases, the parametric maps will be obtained by matching the acquired data to a precomputed dictionary of signal evolutions obtained by means of numerical simulations. For two-dimensional MRF, parameter inference will be performed in two steps [25], where $B_{0}$ values are estimated in the first step and used as input parameter for the second step, where $\mathrm{FF}$, water $\mathrm{T} 1$, and $\mathrm{B}_{1}+$ are obtained. Variation of flip angle and other technical parameters are shown in Fig. 1, while dictionary input parameters are listed in Table 3.

\section{Outcome measures}

Qualitative and quantitative analyses of all collected images will be performed at dedicated workstations (ADW 4.4 and 4.6, General Electric Healthcare, Milwaukee-WI, USA).

Qualitative image analysis Two independent blinded readers (board-certified radiologists with 5- and 15-year experience in abdominal imaging) will assess an image consistency score on a 4-point scale depending on different factors (image signal intensity, imaging resolution, imaging noise, field homogeneity, radiologist's diagnostic confidence in relation to imaging findings), as follows: 1 $=$ poor, insufficient image quality (radiologist not confident at all to propose a diagnosis); 2 = sufficient (it is possible to propose a diagnostic hypothesis but the image quality does not allow a reliable diagnosis); 3 = good (good image quality, which allows to propose a reliable diagnosis); $4=$ excellent (good/excellent image quality with excellent depiction of morphologic details, associated with image characteristics which allows the radiologist to be very confident about the diagnosis).

Quantitative analyses It will require previously defined regions of interest (ROIs) and cannot be assessed in the whole organ/specimen-will be performed on different areas: (a) "targeted tissue" (group B) or "targeted lesion" (groups A and C); (b) tissue close to the targeted ones but above 5-mm distance from the target (non-target tissue). The non-target tissue refers to a parenchymal area not involved in focal alteration/lesion, but with tissue changes related to other systemic diseases such as fibrosis in cirrhotic livers. In groups $\mathrm{A}$ and $\mathrm{C}$, the targeted lesion will be measured including the whole focal lesion, by drawing a hand-made ROI along lesion edges and (when allowed by lesion size) on the lesion central and peripheral zones by circular ROIs. In the latter cases, the area of each ROI will include more than 100 voxels. In order to reduce measurement error, the measurement will be repeated three times, and both single and mean values will be analysed.

Radiological-pathological correlation The same tissue areas will be analysed by the pathologists, thanks to the presence of the anatomical landmarks identified by the radiologist during the MRI examination and referred to

\section{a}
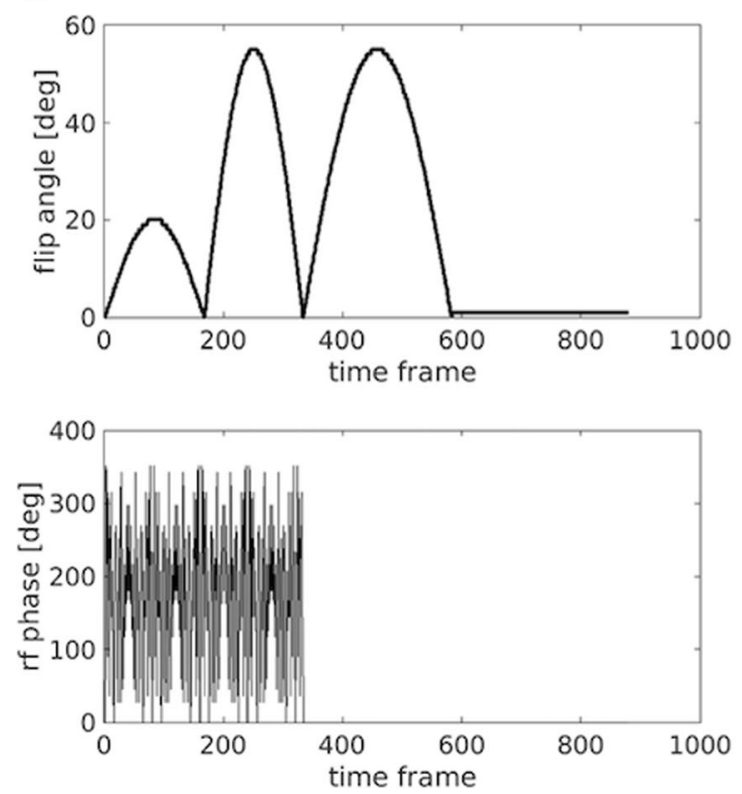

b
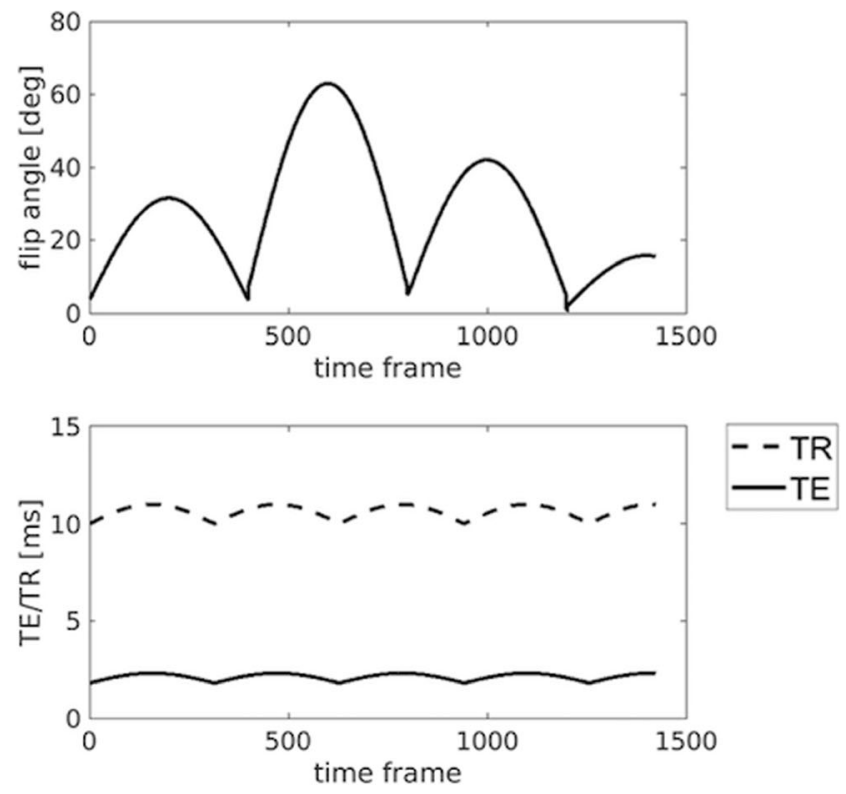

Fig. 1 a Flip angle schedule for three-dimensional magnetic resonance fingerprinting (top row) and radiofrequency phase schedule (bottom row). b Flip angle schedule (top row) and echo time (TE)/repetition time (TR) schedule (bottom row) for two-dimensional magnetic resonance fingerprinting; a radiofrequency ( $\mathrm{r}$ ) spoiling with quadratic phase increment of $117^{\circ}$ is used during the whole acquisition 
Table 3 Values for dictionary creation

\begin{tabular}{lllllll}
\hline & Water T1 $[\mathrm{ms}]$ & Fat T1 [ms] & T2 [ms] & B1+ [a.u.] & B0 [Hz] & Fat fraction \\
\hline Dictionary 1 (2D MRF-step 1) & $0: 300: 4000$ & 500 & NA & $0.1: 0.2: 0.9 ; 0.9: 0.2: 2$ & $-500: 5: 500$ & $0: 0.1: 1$ \\
Dictionary 2 (2D MRF-step 2) & $0: 5: 1550 ; 1600: 100: 4000$ & 500 & NA & $0: 0.1: 2$ & 0 & $0: 0.05: 1$ \\
Dictionary 3 (3D MRF) & 20:10:3000; 3100:50:4500 & NA & $1: 1: 30 ; 35: 5: 50$ & $0: 0.1: 2$ & NA & NA \\
\hline
\end{tabular}

the pathologist. She/he will be able to recognise by using the same section plan defined for imaging and histological analysis. In fact, the section of the twodimensional acquisition plane will be defined together with the pathologists in order to perform the subsequent histological analysis of the same section of the tissue. Unfortunately, the MRI slice thickness will not be the same of the histological slice; as a consequence, the difference in slice thickness will remain a limit of our study. The expected outcomes of radiologicalpathological correlation are summarised in Table 4.

Pathological analysis After the MRI study, two pathologists with 20- and 5-year experience in hepatobiliopancreatic pathologies will analyse all specimens. First, the pathologists will evaluate the whole specimen; then, they will focus on specific tissue slices corresponding to the ones on which the radiologists will provide their diagnosis and diagnostic confidence score.
For group A, gross examination of the liver will be performed reporting on the size and weight of the specimen as well as the appearance of its external surface. In particular, cirrhosis will be described according to the dimension of single nodules as micronodular $(\leq 3 \mathrm{~mm})$ or macronodular $(>3 \mathrm{~cm})$. Subsequently, the liver will be dissected; colour and consistency of liver parenchyma will be described as well as any suspect nodule. Representative samples will be taken for microscopic examination. Main histological features will be reported, and liver neoplasms, when present, will be reported and described according to the 2019 World Health Organization classification of digestive system tumours [26].

For group B, gross examination and dissection will be performed according to the same protocol described for group A. Steatosis will be determined on light microscopy, in formalin-fixed, paraffin-embedded sections and will be described as macrovesicular, in case of hepatocytes distended by a single droplet/few droplets, which

Table 4 Radiological-pathological comparisons and expected outcomes

\begin{tabular}{|c|c|c|c|}
\hline Analysis & Imaging investigated & Pathological reference & Expected outcomes \\
\hline \multicolumn{4}{|l|}{ Qualitative analyses } \\
\hline 1. Image consistency & $\begin{array}{l}\text { CUBE } \\
\text { IDEAL T1w } \\
\text { IDEAL T2w } \\
\text { IDEAL Fat-water } \\
\text { 2D MRCP } \\
\text { 3D MRCP } \\
\text { MP2RAGE } \\
\text { MSE } \\
\text { 3D MRF } \\
\text { 2D MRF }\end{array}$ & $\begin{array}{l}\text { Histological confirmation of the } \\
\text { presence and type of lesion/tissue } \\
\text { alteration identified by imaging }\end{array}$ & $\begin{array}{l}\text { Diagnostic reliability using } \\
\text { morphological sequences }\end{array}$ \\
\hline \multicolumn{4}{|l|}{ Quantitative analyses } \\
\hline $\begin{array}{l}\text { 1. } T 1 \text { relaxation time values of cirrhotic } \\
\text { liver parenchyma } \\
\text { 2. } T 1 \text { relaxation time value of hepatic } \\
\text { lesions } \\
\text { 3. } T 1 \text { relaxation time value of pancreatic } \\
\text { lesion }\end{array}$ & $\begin{array}{l}\text { MP2RAGE } \\
\text { 3D MRF (T1-map) } \\
\text { 2D MRF (T1-map) }\end{array}$ & $\begin{array}{l}\text { 1. Fibrotic/regenerative characteristics } \\
\text { of the liver tissue } \\
\text { 2. Focal lesions affecting the cirrhotic } \\
\text { liver } \\
\text { 3. Pancreatic lesion characteristics }\end{array}$ & $\begin{array}{l}\text { 1. Correlation between the } \\
\text { fibrotic degree and the T1-value } \\
\text { 2. Differential diagnosis of the } \\
\text { focal hepatic lesions based on } \\
\text { T1-value } \\
\text { 3. Pancreatic lesion stroma related } \\
\text { to T1-value }\end{array}$ \\
\hline $\begin{array}{l}\text { 1. T2 relaxation time values of hepatic } \\
\text { lesions } \\
\text { 2. } T 2 \text { relaxation time value of pancreatic } \\
\text { lesion }\end{array}$ & $\begin{array}{l}\text { MSE } \\
\text { 3D MRF (T2-map) }\end{array}$ & $\begin{array}{l}\text { 1. Focal lesions affecting the cirrhotic } \\
\text { liver } \\
\text { 2. Pancreatic lesion characteristics }\end{array}$ & $\begin{array}{l}\text { 1. Differential diagnosis of the } \\
\text { focal hepatic lesions based on } \\
\text { T2-value } \\
\text { 2. Pancreatic lesion aggressiveness } \\
\text { related to T2-value }\end{array}$ \\
\hline $\begin{array}{l}\text { Fat-fraction values of steatotic liver } \\
\text { parenchyma }\end{array}$ & $\begin{array}{l}\text { IDEAL Fat-water } \\
\text { 2D MRF (fat-fraction and } \\
\text { water-fraction) } \\
3 \text { D MRF (fat-fraction and } \\
\text { water-fraction) }\end{array}$ & $\begin{array}{l}\text { Well-established histological } \\
\text { classification of the steatosis } \\
\text { distribution }\end{array}$ & $\begin{array}{l}\text { Good radiological-pathological } \\
\text { correlation about the degree } \\
\text { of steatosis }\end{array}$ \\
\hline
\end{tabular}


displace the nucleus, or microvesicular, in case of swollen hepatocytes containing very small vacuoles, without displacement of the nucleus. In addition, the percentage of hepatocytes with steatosis as well as their localisation (pericentral, lobular, periportal) will be reported.

For group $\mathrm{C}$, before sampling the specimen, a multicoloured inking of all the pancreatic circumferential margins will be performed. The pancreatic head will be sliced along the axial plane, perpendicularly to the descending part of the duodenum, with a dissection plane similar to that of MRI. Dissection will include the entire head of the pancreas and any adherent structures such as duodenal wall, distal common bile duct, and vascular resection, when present. All main histological features of the tumour and collateral tissue will be reported during the microscopic examination. Margins will be defined negative when no tumour cells are found within $1 \mathrm{~mm}$ from inked margins [27].

\section{Sample size and statistical analysis}

To the best of our knowledge, the EXLIPSE study could be the first study on quantitative 7-T MRI of ex vivo human liver and pancreatic specimens. As a consequence, the sample size calculation cannot be based on previous published experiences. Thus, this preliminary study will start by including at least ten ex vivo specimens per group, a sample size considered as appropriate in terms of feasibility assessment.

Before performing inferential tests, KolmogorovSmirnov's test will be used to evaluate data distribution. In the case of normal distribution, the quantitative T1 and T2 values inside the ROIs obtained will be compared with the different degree assigned to the examined histological tissue characteristics (fibrotic grade, fat content, and pancreatic cancer marker of aggressiveness, such as involvement of circumferential resection margins) using a mixed effects ANOVA model. By this statistics, it will be possible to identify differences among the different groups. In the case of not normal distribution, they will be compared by using the Kruskal-Wallis test. To assess the correlation between the relaxation time values and histological measurements Pearson $r$ or Spearman $\rho$ will be applied, according to data distribution. When the ANOVA test includes several groups and the analysis results are significant, a post hoc analysis will be performed applying the Bonferroni correction

The $p$ values will be calculated using the Chi-square distribution for nominal variables. Thus, the correlation between the morphological MRI data and the information provided by the macroscopic and microscopic histological examination of the specimens will be performed. Specimen characteristics will be summarised using descriptive statistics: mean and standard deviation, or median and range for continuous variables, in the case of normal or not normal distribution, respectively, absolute and percentage frequencies for categorical variables.

A $p$ value lower than 0.05 will be considered statistically significant, with exception of cases when Bonferroni correction will be appropriate.

\section{Discussion}

The use of specimens allows a high spatial resolution without motion artefacts to guarantee a precise comparison between the information obtained by image analysis and the specimen macroscopic and microscopic pathological examinations. The effort made to build this study protocol was driven by the belief that ex vivo specimens investigated by 7-T MRI allow a reliable comparison between radiological images and histology. Moreover, 7-T MRI has already been demonstrated as a useful tool able to provide information on surgical margins after surgery. In particular, 7-T MRI showed a good correlation with pathology in the evaluation of negative surgical margins after partial nephrectomy with and sensitivity and specificity for MR assessment of negative surgical margins of $100 \%$ and $75 \%$, respectively. With a mandatory protocol optimisation to minimise the scan time, the authors proposed the 7-T MRI specimen evaluation as a promising intraoperative tool to improve the surgical outcomes about margins and parenchymal sparing [28]. As already demonstrated in other anatomical districts, the comparison between 7-T MRI and pathology might identify previously undefined quantitative imaging biomarkers and might recognise morphological changes able to give information for the diagnosis and management of many systemic (cirrhosis and steatosis) and focal hepatic diseases, in particular cancer (PDAC and hepatocellular carcinoma).

Due to the small study sample per group, the identified imaging biomarkers and morphological tissue changes will have to be validated by future dedicated studies, which will be conducted both on a larger ex vivo sample and on an in vivo population.

This research protocol had to overcome some difficulties in its design. First, specimen storage: the pathologist responsible for the analysis of liver and pancreas specimens was asked to evaluate how to store the specimens before histological analysis. The specimens should not be treated with formalin before 7-T MRI because a decrease in magnetic resonance tissue characteristics including $\mathrm{T} 1$ and T2 relaxation times have been demonstrated after fixation [22, 29-31].

Thus, the pathologists assessed that no tissue damage occurs if the time length from surgical resection to MRI exam and to delivery to the pathologist is less than $24 \mathrm{~h}$. During these $24 \mathrm{~h}$, the specimen should be maintained at a temperature of $4{ }^{\circ} \mathrm{C}$, with the exception of the time spent in the MRI magnet when the specimen temperature will 
arise above $15^{\circ} \mathrm{C}$. This temperature will be controlled during the exam so that the heating related to radiofrequency exposure does not damage the tissue. The exam will be interrupted if the temperature exceeds $25^{\circ} \mathrm{C}$. Second, the logistics: the lab where the 7-T device is located is about $15 \mathrm{~km}$ from the surgical suites and from pathology department. For this reason, the transportation of the specimens required dedicated medical vehicles. Finally, the lack of previous similar experiences in the literature: the 7-T knee coil has been predominantly used and optimised for knee investigations; thus, no examples of technical parameters to study ex vivo specimens were available. As a consequence, before starting the EXLIPSE study, an acquisition protocol had to be set-up.

This study will provide the possibility to improve our knowledge about the quantitative abdominal imaging assessment. In fact, the EXLIPSE study could represent an important step to further develop MRF sequences on 3-T MRI. The advantage of using 7-T radiological-pathological correlation to optimise MRF consists in the opportunity of employing the tissue morphologic and quantitative characteristics discovered by the ultrahigh field and confirmed by histology as a reference standard for constructing the signal on the similar sequences for 3-T systems. We expect that the higher 7- $\mathrm{T}$ signal in the quantitative maps could provide a useful information, even if for relatively small samples. The higher resolution could provide a more suitable correlation with micron-size histology evaluation.

Studies at lower field strengths could benefit from larger samples to achieve further results. Once imaging biomarkers are identified, the next step will be to exploit this knowledge for application on 3-T device, to create a more widespread demonstration and application of their clinical utility, due to the remarkably larger availability of the 3-T scanner. Protocol and sequence optimisation process will be mandatory at $3 \mathrm{~T}$ due to the different contrast (increased $\mathrm{T} 1$ and decreased $\mathrm{T} 2$ at $7 \mathrm{~T}$ than at $3 \mathrm{~T}$ ), potential artefacts observed on the images obtained at different static magnetic fields, and the different availability of dedicated coils. However, the presence of well-optimised dedicated coils for abdominal imaging at $3 \mathrm{~T}$ may lead to a more rapid optimisation of sequences and protocols.

\section{Supplementary information}

Supplementary information accompanies this paper at https://doi.org/10. 1186/s41747-020-00185-y.

Additional file 1.

\section{Abbreviations}

ANOVA: Analysis of variance; IDEAL: Iterative decomposition of water and fat with echo asymmetry and least-squares estimation; MRF: Magnetic resonance fingerprinting; MRI: Magnetic resonance imaging; PDAC: Pancreatic ductal adenocarcinoma; ROI: Region of interest

\section{Acknowledgements}

We thank the IMAGO7 Foundation for their scientific support.

\section{Authors' contributions}

Prof. LC and Dr. RC conceived of the presented idea. Dr. MC and Dr. GB developed the quantitative sequences which will be applied in the study, and they will perform the post-processing computations. Prof. MT will verify the consistency of the quantitative sequences and the analytical methods. Prof. PDS and Prof. UB will contribute to organ sample preparation. Prof. DC and Dr. ACl will perform the histological analysis of all specimens. Dr. FC and Dr. GA will contribute in carrying out the experiment, either with the imaging acquisition protocol or with the morphological images reviewing in order to score the image quality and the diagnostic confidence. Dr. RC will write the manuscript. Prof. LC will supervise the findings of this work and the development of the manuscript. All authors will discuss the results and contribute to the final manuscript.

Funding

None

\section{Availability of data and materials}

The datasets used and/or analysed during the current study are available from the corresponding author on reasonable request.

\section{Ethics approval and consent to participate}

The study will be performed in agreement with the dictates of the Helsinki Declaration and the rules of Good Clinical Practice (E6: Good Clinical Practice: Consolidated Guideline (CPMP/ICH/135/95)). The permission of the Ethical Committee is wanted and the request to conduct a prospective study is sent to the Institutional Authority (protocol number approval: 13646). Before starting the study, every participant will be accurately informed about the management of the resected specimen and will be given the informative letter for them and for their general practitioner, the forms of the informed consent, and the one for the treatment of their clinical data for research purposes. All participants will be registered after signing the informed consent. The informed consent to participate to the "EXLIPSE" research study does not apply to group B since the authorisation to organ donation in our region implies also the consent to investigate the explanted organs for scientific purposes (Tuscany Transplant Organization, OTT/SR/001.HB).

\section{Consent for publication}

Included in consent to participate to the study

\section{Competing interests}

None

\section{Author details}

${ }^{1}$ Division of Diagnostic and Interventional Radiology, University of Pisa, Via Paradisa, 2 - Cisanello Hospital, 56100 Pisa, Italy. ${ }^{2}$ MMAGO7 Foundation IRCCS Stella Maris, Pisa, Italy. ${ }^{3}$ Division of Pathology, University of Pisa, Pisa, Italy. ${ }^{4}$ Division of Hepatic Surgery and Liver Transplant, University of Pisa, Pisa, Italy. ${ }^{5}$ Division of General and Transplant and Surgery, University of Pisa, Pisa, Italy.

Received: 15 April 2020 Accepted: 3 September 2020

Published online: 15 October 2020

\section{References}

1. Kraff O, Fischer A, Nagel AM, Mönninghoff C, Ladd ME (2015) MRI at 7 Tesla and above: demonstrated and potential capabilities. J Magn Reson Imaging 41:13-33. https://doi.org/10.1002/jmri.24573

2. Pohmann R, Speck O, Scheffler K (2016) Signal-to-noise ratio and MR tissue parameters in human brain imaging at 3, 7, and 9.4 tesla using current receive coil arrays. Magn Reson Med 75:801-809. https://doi.org/10.1002/ mrm.25677

3. Ma D, Gulani V, Seiberlich $N$ et al (2013) Magnetic resonance fingerprinting. Nature 495:187-192. https://doi.org/10.1038/nature11971

4. Chen $Y$, Jiang $Y$, Pahwa $S$ et al (2016) MR fingerprinting for rapid quantitative abdominal imaging. Radiology 279:278-286. https://doi.org/10. 1148/radiol.2016152037 
5. Avanzo M, Stancanello J, El Naqa I (2017) Beyond imaging: the promise of radiomics. Phys Med 38:122-139. https://doi.org/10.1016/j.ejmp.2017.05.071

6. Dekkers IA, Lamb HJ (2018) Clinical application and technical considerations of T1 and T2 $(*)$ mapping in cardiac, liver, and renal imaging. Br J Radiol 91: 1-13. https://doi.org/10.1259/bjr.20170825

7. Petitclerc L, Gilbert G, Nguyen BN, Tang A (2017) Liver fibrosis quantification by magnetic resonance imaging. Top Magn Reson Imaging 26:229-241. https://doi.org/10.1097/RMR.0000000000000149

8. Donato H, França M, Candelária I, Caseiro-Alves F (2017) Liver MRI: from basic protocol to advanced techniques. Eur J Radiol 93:30-39. https://doi. org/10.1016/j.ejrad.2017.05.028

9. Caussy C, Reeder SB, Sirlin CB, Loomba R (2018) Noninvasive, quantitative assessment of liver fat by MRI-PDFF as an endpoint in NASH trials. Hepatology 68:763-772. https://doi.org/10.1002/hep.29797

10. Chouhan MD, Firmin L, Read S, Amin Z, Taylor SA (2019) Quantitative pancreatic MRI: a pathology-based review. Br J Radiol 92:1-10. https://doi. org/10.1259/bjr.20180941

11. Dhir M, Lyden ER, Smith LM, Are C (2012) Comparison of outcomes of transplantation and resection in patients with early hepatocellular carcinoma: a meta-analysis. HPB (Oxford) 14:635-645. https://doi.org/10. 1111/j.1477-2574.2012.00500.x

12. Lim KB, Schiano TD (2012) Long-term outcome after liver transplantation. Mt Sinai J Med 79:169-189. https://doi.org/10.1002/msj.21302

13. Mehta N, Dodge JL, Grab JD, Yao FY (2020) National experience on downstaging of hepatocellular carcinoma before liver transplant: influence of tumor burden, alpha-fetoprotein, and wait time. Hepatology 71:943-954. https://doi.org/10.1002/hep.30879

14. de Graaf EL, Kench J, Dilworth P et al (2012) Grade of deceased donor liver macrovesicular steatosis impacts graft and recipient outcomes more than the donor risk index. J Gastroenterol Hepatol 27:540-546. https://doi.org/10. 1111/j.1440-1746.2011.06844.x

15. Nafidi O, Marleau D, Roy A, Bilodeau M (2010) Identification of new donor variables associated with graft survival in a single-center liver transplant cohort. Liver Transpl 16:1393-1399. https://doi.org/10.1002/lt.22176

16. McCormack L, Dutkowski P, El-Badry AM, Clavien PA (2011) Liver transplantation using fatty livers: always feasible? J Hepatol 54:1055-1062. https://doi.org/10.1016/j.jhep.2010.11.004

17. Durand F, Renz JF, Alkofer B et al (2008) Report of the Paris consensus meeting on expanded criteria donors in liver transplantation. Liver Transpl 14:1694-1707. https://doi.org/10.1002/lt.21668

18. Lehmann A, Denkert C, Budczies J, et al (2009) High class I HDAC activity and expression are associated with ReIA/p65 activation in pancreatic cancer in vitro and in vivo. BMC Cancer 2009 9:395. doi.https://doi.org/10.1186/ 1471-2407-9-395

19. Loos M, Hedderich DM, Ottenhausen M et al (2009) Expression of the costimulatory molecule B7-H3 is associated with prolonged survival in human pancreatic cancer. BMC Cancer 9:463. https://doi.org/10.1186/14712407-9-463

20. Kleiner DE, Brunt EM, Van Natta M, et al (2005) Nonalcoholic Steatohepatitis Clinical Research Network. Design and validation of a histological scoring system for nonalcoholic fatty liver disease. Hepatology 41:1313-1321. doi. https://doi.org/10.1002/hep.20701

21. Shepherd TM, Thelwall PE, Stanisz GJ, Blackband SJ (2009) Aldehyde fixative solutions alter the water relaxation and diffusion properties of nervous tissue. Magn Reson Med 62:26-34. https://doi.org/10.1002/mrm.21977

22. Yong-Hing CJ, Obenaus A, Stryker R, Tong K, Sarty GE (2005) Magnetic resonance imaging and mathematical modeling of progressive formalin fixation of the human brain. Magn Reson Med 54:324-332. https://doi.org/ 10.1002/mrm.20578

23. Reeder SB, Pineda AR, Wen Z et al (2005) Iterative decomposition of water and fat with echo asymmetry and least-squares estimation (IDEAL): application with fast spin-echo imaging. Magn Reson Med 54:636-644. https://doi.org/10.1002/mrm.20624

24. Marques JP, Kober T, Krueger G, van der Zwaag W, Van de Moortele PF, Gruetter R (2010) MP2RAGE, a self bias-field corrected sequence for improved segmentation and T1-mapping at high field. Neuroimage 49: 1271-1281. https://doi.org/10.1016/j.neuroimage.2009.10.002

25. Cencini M, Biagi L, Kaggie JD, Schulte RF, Tosetti M, Buonincontri G (2019) Magnetic resonance fingerprinting with dictionary-based fat and water separation (DBFW MRF): a multi-component approach. Magn Reson Med 81 3032-3045. https://doi.org/10.1002/mrm.27628
26. Nagtegaal ID, Odze RD, Klimstra D et al (2020) WHO Classification of Tumours Editorial Board. The 2019 WHO classification of tumours of the digestive system. Histopathology 76:182-188. https://doi.org/10.1111/his. 13975

27. Verbeke CS, Leitch D, Menon KV, McMahon MJ, Guillou PJ, Anthoney A (2009) Redefining the R1 resection in pancreatic cancer. Br J Surg 93:12321237. https://doi.org/10.1002/bjs.5397

28. Van Oostenbrugge TJ, Runneboom W, Bekers E et al (2019) MRI as a tool to assess surgical margins and pseudocapsule features directly following partial nephrectomy for small renal masses. Eur Radiol 29:509-516. https:// doi.org/10.1007/s00330-018-5630-9

29. Wang C, Song L, Zhang R, Gao F (2018) Impact of fixation, coil, and number of excitations on diffusion tensor imaging of rat brains at 7.0 T. Eur Radiol Exp 2:25. https://doi.org/10.1186/s41747-018-0057-2

30. Purea A, Webb AG (2006) Reversible and irreversible effects of chemical fixation on the NMR properties of single cells. Magn Reson Med 56:927-931. https://doi.org/10.1002/mrm.21018

31. Raman MR, Shu Y, Lesnick TG, Jack CR, Kantarci K (2017) Regional T1 relaxation time constants in ex vivo human brain: longitudinal effects of formalin exposure. Magn Reson Med 77:774-778. https://doi.org/10.1002/ mrm.26140

\section{Publisher's Note}

Springer Nature remains neutral with regard to jurisdictional claims in published maps and institutional affiliations.

\section{Submit your manuscript to a SpringerOpen ${ }^{\circ}$ journal and benefit from:}

- Convenient online submission

- Rigorous peer review

- Open access: articles freely available online

High visibility within the field

- Retaining the copyright to your article

Submit your next manuscript at $>$ springeropen.com 